\title{
Model-based Halftoning for Color Image Segmentation
}

\author{
Jan Puzicha and Serge Belongie \\ UC Berkeley, Dept. Computer Science \\ \{puzicha,sjb\}@cs.berkeley.edu
}

\begin{abstract}
Grouping algorithms based on histograms over measured image features have very successfully been applied to textured image segmentation $[2,11,6]$. However, the competing goals of statistical estimation significance demanding few quantization levels versus the necessary richness in representation often prevent a successful application for the color cue, since quantization may result in contouring.

In this paper, we combine a novel halftoning technique called spatial quantization with distribution-based grouping algorithms to synthesize a powerful color image segmentation technique. The spatial quantization simultaneously determines color palette and halftoning by optimizing a joint cost function. It therefore allows for a highly adapted image representation with a smooth transition of color distributions for non-constant image surfaces.
\end{abstract}

\section{Introduction}

In recent years, a major paradigm shift can be observed in low-level computer vision: Instead of representing measurements as vectors in some feature space, the distribution over measured features provides a powerful representation overcoming some of the key difficulties of feature vector based approaches. Similarity measures between empirical histograms have played a major role in the development of example-based image retrieval $[1,8]$. However, distribution-based similarity functions turned out to be very successful in recognition [10], classification [7], boundary detection [9] and image segmentation [2, 11, 6].

To be applicable, a sufficient number of measurements must be available in order to provide enough information for a statistically significant estimate of a feature histogram. Especially in edge detection and segmentation, the necessary localization and thus the sparseness of available data demands as few signal quantization levels as possible. In [9], the quantization has been adapted for each single image pixel to devise a novel color edge detection technique. However, in image segmentation the binning must be kept

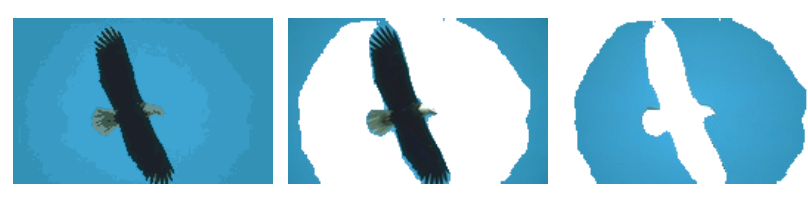

Figure 1. Example of a contouring effect.

constant over the entire image. Especially for color, the resulting contouring effects provide a major obstacle for a successful application of distribution-based techniques, see Fig. 1 for an example. Here, the left image displays a quantization into 8 colors and the right two images depict a segmentation result for $L=2$ segments employing the histogram grouping technique of [6]. The sky is quantized into rings of constant color, resulting in maximally different histograms when comparing different parts of the sky.

While digital halftoning has hardly ever been considered as a useful computer vision technique, the ability to avoid contouring and thus to preserve the visual perception of an image despite coarse quantization renders it a highly attractive method for preprocessing in image segmentation. In this paper, we develop a novel halftoning technique called spatial quantization that simultaneously determines color palette and halftoning by optimizing a joint cost function [5]. As we will demonstrate it allows for a highly adapted image representation with a smooth transition of color distributions for non-constant image surfaces even for very few quantization levels. Its usefulness as a preprocessing technique is shown for two different grouping algorithms, namely histogram grouping [6] and the normalized cut [11]. This indicates its potential to compute a generic image representation independent of the chosen image segmentation algorithm.

\section{Model-based Halftoning}

Digital Halftoning refers to a class of techniques developed to reduce the visual disturbance that is observed in quantized color images. Color reduction is thus usually implemented in a sequential two-step procedure. First, 
a pixel-wise clustering scheme is used for quantization, e.g. median cut or $K$-means. Then a halftoning technique like error diffusion, dithering or model-based halftoning is applied employing the spatial averaging property of human perception to create extra illusory colors by spatial mixing. See [3] for an overview and references.

It should be noted that this sequential process is suboptimal in that different criteria are optimized. To overcome this deficiency, we recently developed a novel cost function based approach called spatial quantization to simultaneously optimize quantization and halftoning [5]. As the key algorithmic idea, spatial quantization aims at minimizing the difference between the perceived image before and after quantization, employing a linear model of human color perception. It thereby allocates a color palette that is optimally tuned to the halftoning process.

More formally, suppose that a model of human perception is given by a discrete kernel $W_{k}$, where $k \in\{1,2,3\}$ denotes the color coordinate in CIE $L^{*} a^{*} b^{*}$ color space ${ }^{1}$. In the experiments, we used a simple Gaussian kernel of identical standard deviation $\sigma$ for all channels as an impulse response function, but several more elaborated perception models have been proposed, see [5] for a discussion and references. Denote by $I_{k}$ the $k$-th color palette of an input image 1 . Then the $k$-th color plane of the perceived image $P_{k}$ is modeled by

$$
P_{k}(I)=W_{k} * I_{k}
$$

Now introduce a color palette of quantized colors $\mathbf{Y}=$ $\left(\mathbf{y}_{\nu}^{t}\right)_{\nu=1, \ldots, K}, \mathbf{y}_{\nu} \in \mathbb{R}^{3}$, where the superscript $t$ denotes the transpose of a vector. A quantization is defined as an assignment of pixels to quantized colors, which is formalized by Boolean assignment variables $M_{i \nu} \in\{0,1\}$. Here, $M_{i \nu}=1$ codes that the image site $i$ is quantized to color $\mathbf{y}_{\nu}$. All assignments are summarized in terms of a Boolean assignment matrix $\mathbf{M} \in \mathcal{M}$, where

$$
\mathcal{M}=\left\{\mathbf{M} \in\{0,1\}^{N \times K}: \sum_{\nu=1}^{K} M_{i \nu}=1, \forall i\right\} .
$$

The quantized image is now formally obtained by the matrix multiplication MY. As a cost function for faithful color reproduction, we employ the sum of the pixel-wise square distance between the perceived image before and after quantization,

$$
\mathcal{H}(\mathbf{M}, \mathbf{Y})=\sum_{k=1}^{3}\left\|P_{k}(I)-P_{k}(\mathbf{M} \mathbf{Y})\right\|^{2}
$$

This model generalizes the classical $K$-means cost function, which is obtained for a Dirac kernel $W_{k}$, i.e. an undistorted perception. The optimal quantized image is obtained

\footnotetext{
${ }^{1}$ It is important to choose a uniform color space to justify the model of illusory colors by linear superposition.
}

by minimizing (3) w.r.t. $\mathbf{Y}$ and $\mathbf{M}$, i.e. color palette and quantization are obtained simultaneously. Eq. (3) turns out to be fully quadratic in both $\mathbf{y}_{\nu}$ and $M_{i \nu}$ and poses a hard and very large mixed combinatorial optimization problem. We developed a highly efficient multiscale optimization scheme, the presentation of which is beyond the scope of this paper. We refer to [5] for details where it has also been demonstrated that spatial quantization dramatically increases performance compared to standard color reduction algorithms.

\section{Distribution-based Image Segmentation}

As the key component of our segmentation approach, the input image is represented by the local color distribution around a pixel. More formally, denote by $\mathbf{M}$ again the assignment matrix of the quantized image and let $\mathcal{W}_{i}$ denote a local neighborhood or window around pixel $i$. Then

$$
h_{i}(\nu)=\frac{\sum_{j \in \mathcal{W}_{i}} M_{j \nu}}{\left|\mathcal{W}_{i}\right|}
$$

computes the local color histogram in window $\mathcal{W}_{i}$. Eq. (4) can be interpreted as an adaptive distribution estimate, where the bin centers $\mathbf{Y}_{\nu}$ are adapted to the specific input image at hand.

Next, we briefly recapitulate two recently introduced grouping techniques which employ the image representation (4) to compute segmentations. Histogram grouping [6], also referred to as the asymmetric clustering model (ACM), allows us to group histograms $h_{i}(\nu)$ directly. It is based on a statistical mixture model with $L$ prototypical distributions $h_{p}(\nu)$. To be specific, denote by the Boolean entities $P_{i p} \in\{0,1\}$ the (unobserved) assignment of the empirical distribution $h_{i}(\nu)$ to one out of the $L$ prototypes $h_{p}(\nu)$ modeling the color distribution of the complete segment $p$. An image segmentation is computed by maximizing the $\log -$ likelihood of the model

$$
\mathcal{L}\left(\mathbf{P}, h_{p}\right)=\sum_{i} \sum_{p=1}^{L} P_{i p} \sum_{\nu=1}^{K} h_{i}(\nu) \log h_{p}(\nu)
$$

w.r.t. the discrete $P_{i p}$ and the continuous parameters $h_{p}(\nu) \in[0,1]$. Introducing Lagrange parameters for the normalizing constraints $\sum_{\nu} h_{p}(\nu)=1$ we obtain the following stationary equations by differentiation,

$$
\begin{aligned}
h_{p}(\nu) & =\sum_{i} \frac{P_{i p}}{\sum_{j} P_{j p}} h_{i}(\nu), \\
P_{i p} & =\arg \min _{p^{*}} \sum_{\nu} h_{i}(\nu) \log h_{p^{*}}(\nu) .
\end{aligned}
$$

This system of equations closely resembles the $K$-means algorithm for vectorial clustering, where (6) corresponds to 
the centroid equation, now for histograms, and (7) implements a nearest neighbor rule, replacing the usual Euclidean metric by a more appropriate KL-divergence measure [6]. The optimal solution is then iteratively determined ${ }^{2}$. The ACM model is easy to implement and allows us to compute image segmentations in a few seconds. However, while robust to some variations it models image segments by constant color distributions and will thus inevitably fail on smoothly varying segments like ramps.

The normalized cut [11] or NCut is a more indirect segmentation scheme in that the histograms are first compared to produce pairwise similarity scores $W_{i j}$ by computing the $\chi^{2}$ test statistic between the histograms $h_{i}(\nu)$ and $h_{j}(\nu)$ at image sites $i$ and $j$, respectively, and defining $W_{i j}=\exp \left(-\chi^{2} / \sigma\right)$. As shown in [11], a good approximation to the optimal segmentation can be computed via spectral bisection on the normalized Laplacian of the weighted graph. ${ }^{3}$ This is done by thresholding the eigenvector corresponding to the second smallest eigenvalue in the generalized eigenvalue problem

$$
(\mathbf{D}-\mathbf{W}) \mathbf{y}=\lambda \mathbf{D} \mathbf{y}
$$

where $D_{i i}=\sum_{j} W_{i j}$. Because connections are only allowed between nearby sites in the image, $\mathbf{W}$ is typically quite sparse. This characteristic of the NCut approach not only permits an efficient solution, but also leads to proper grouping of smoothly varying regions since similarity is propagated through local connections.

While our normalized cut implementation is substantially slower than $\mathrm{ACM}^{4}$ its modeling assumptions are less restrictive. In particular, it achieves better results for smoothly varying segments and allows one to integrate multiple cues into the definition of $W_{i j}$ [4].

\section{Results}

For the experiments we selected images from the Corel database that are simple in the sense that most human observers would agree upon an image segmentation solution with $L=2$ segments. However, the segments themselves still exhibit substantial variability and therefore pose a challenge for an automatic segmentation system.

For all experiments, we employed $K=8$ quantization levels and a window size of $7 \times 7$. The NCut partitions were found on a half-resolution grid, with a run-time of about 1.5 minutes using the eigs.m function in Matlab.

\footnotetext{
${ }^{2}$ In [6], we devised a multiscale optimization scheme for accelerated maximization.

${ }^{3}$ This process can be run recursively for segmentation into multiple groups, but for the present comparison we only concern ourselves with the first partition.

${ }^{4}$ About a factor 50 in terms of actual run-time. However, normalized cut is partially implemented in Mat lab while ACM is completely written in $\mathrm{C}++$ complicating a fair comparison.
}
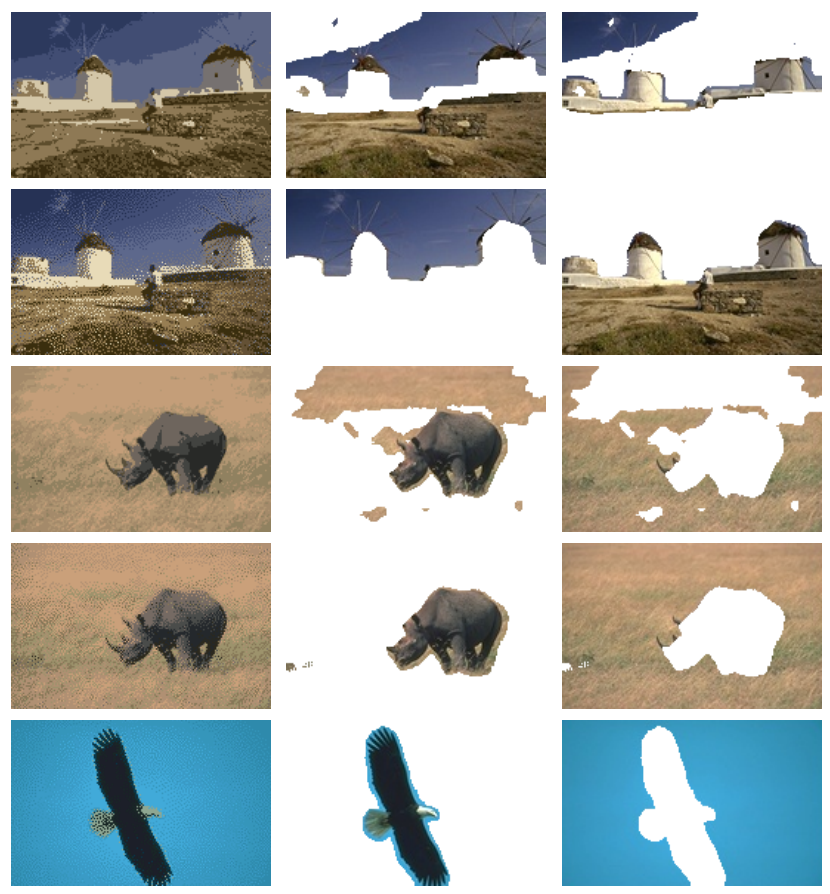

Figure 2. Results with ACM for $L=2$. Rows 1, 3: $K$-means, rows 2, 4, 5: spatial quantization.

In Fig. 3 we compare the segmentation results obtained by ACM for different image quantization schemes, (a) the novel spatial quantization scheme, (b) a $K$-means based quantization and (c) a sequential color reduction by $K-$ means followed by a halftoning step ${ }^{5}$. It can be seen that a $K$-means based image quantization leads to severe contouring and consequently to bad segmentation results. A subsequent halftoning step somewhat dimishes the problem, but errors remain, while only spatial quantization makes use of the full potential of the halftoning idea leading to excellent segmentation results. As seen from Fig. 3, the visual quality of the quantized images obeys the same rank order, where spatial quantization nicely preserves the color content of the image. The results are confirmed by the experiments depicted in Fig. $2^{6}$.

In Fig. 4 example results for the normalized cut are depicted. The figure demonstrates the shortcoming of ACM, i.e. the assumption of constant color distribution. It should be emphasized that the segmentation errors for ACM are no longer due to the shortcomings of the representation. Con-

\footnotetext{
${ }^{5}$ For halftoning, we optimized (3) with respect to $\mathbf{M}$ while keeping the color palette $\mathbf{Y}$ fixed.

${ }^{6}$ The halftoning results are not depicted due to limitations in space, but are very similar to the $K$-means results, emphasizing the necessity of full spatial quantization.
} 

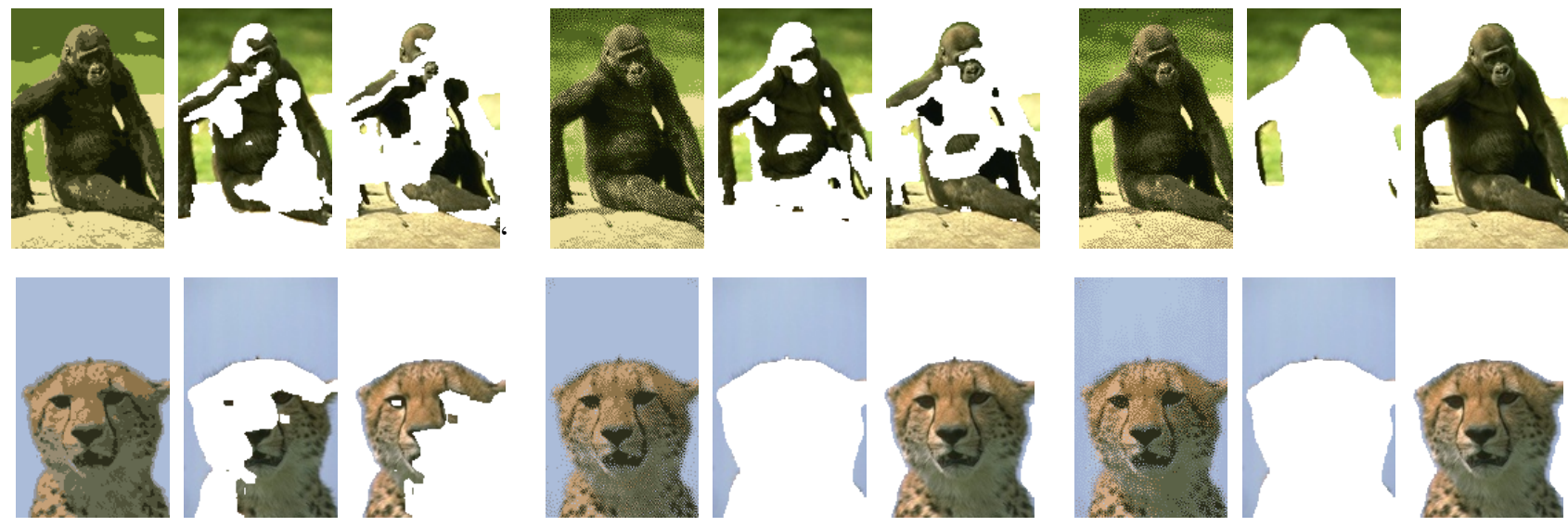

Figure 3. Results obtained by ACM for $L=2$. Left: $K$-means quantization, middle: halftoning, right: spatial quantization. See Fig. 1 for the $K$-means result of the eagle image.

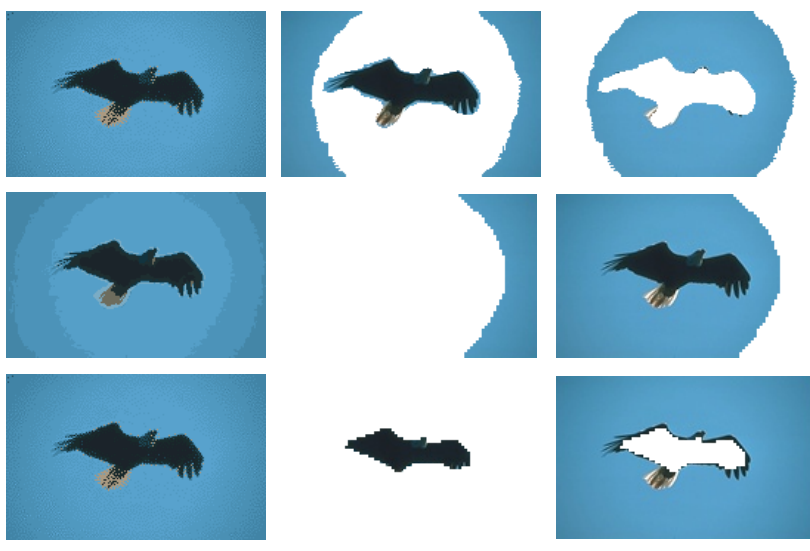

Figure 4. Results for $L=2$. 1st row: $\mathbf{S Q}+$ ACM, 2nd row: $K$-means + NCut, 3rd row: $S Q+$ NCut.

sequently, the normalized cut enables us to compute a very good segmentation solution in this case.

\section{Conclusion}

As the main contribution of this paper, we proposed to employ digital halftoning to compute image representations that are optimally suited for colored image segmentation. We presented a novel color quantization scheme, that jointly optimizes color palette selection and halftoning and thus allows for an optimal adaptation to the image. The resulting image representation allows improved performance for a large class of segmentation algorithms, where exemplary results have been shown for histogram grouping and the normalized cut.

Acknowledgement: This research has been funded by the German Research Foundation (DFG) under grant PU-165/1, by the NSF under grant IRI-94-11334 and by an NSF Graduate Fellowship.

\section{References}

[1] M. Flickner et. al. Query by image and video content: The QBIC system. IEEE Computer, pages 23-32, Sept. 1995.

[2] T. Hofmann, J. Puzicha, and J. Buhmann. Unsupervised texture segmentation in a deterministic annealing framework. IEEE PAMI, 20(8):803-818, 1998.

[3] H. Kang. Digital Color Halftoning. IEEE Press, 1999.

[4] J. Malik, S. Belongie, J. Shi, and T. Leung. Textons, contours and regions: Cue integration in image segmentation. In Proc. ICCV, pages 918-925, 1999.

[5] J. Puzicha, M. Held, J. Ketterer, J. Buhmann, and D. Fellner. On spatial quantization of color images. IEEE IP, 2000.

[6] J. Puzicha, T. Hofmann, and J. Buhmann. Histogram clustering for unsupervised segmentation and image retrieval. Pattern Recognition Letters, 20(9):899-909, 1999.

[7] J. Puzicha, Y. Rubner, C. Tomasi, and J. Buhmann. Empirical evaluation of dissimilarity measures for color and texture. In Proc. ICCV, pages 1165-1173, 1999.

[8] Y. Rubner and C. Tomasi. A metric for distributions with applications to image databases. In Proc. ICCV, pages 5966, 1998.

[9] M. Ruzon and C. Tomasi. Color edge detection with the compass operator. In Proc. CVPR, pages 160-166, 1999.

[10] B. Schiele and J. Crowley. Recognition without correspondence using multidimensional receptive field histograms. International Journal of Computer Vision (to appear), 2000.

[11] J. Shi and J. Malik. Normalized cuts and image segmentation. In Proc. CVPR, pages 731-737, 1997. 